\title{
Pediatric supratentorial high-grade gliomas
}

\author{
Mandeep S. Tamber, M.D., and James T. Rutka, M.D., Ph.D., F.R.C.S.(C) \\ Division of Neurosurgery, The Hospital for Sick Children, University of Toronto, Toronto, Ontario, \\ Canada
}

\begin{abstract}
The purpose of this review is to highlight some of the pertinent concepts and controversies surrounding the diagnosis and treatment of pediatric supratentorial high-grade gliomas. Unlike the adult counterparts, pediatric high-grade gliomas are likely derived from distinct cytogenetic and molecular alterations. Surgery has been shown to play a role in extending patient survival. Some success is associated with the provision of chemotherapy. Radiotherapy remains an important adjunct in children older than age 3 years. The challenges involved in improving the poor prognosis of children in whom these very aggressive tumors have been diagnosed will be discussed, as well as some of the novel approaches being investigated to improve patient survival and quality of life.
\end{abstract}

\section{KEY WORDS • high-grade glioma • supratentorial tumor • pediatric neurosurgery • outcome}

A growing body of evidence suggests that high-grade gliomas in pediatric patients manifest properties that clearly distinguish them from their adult counterparts. Some of the most striking differences between them lie in their differential response to conventional treatment modalities. Whereas adjuvant chemotherapy has been shown to exert only a modest survival benefit when undertaken in adult patients, chemotherapy combined with surgery and radiotherapy is associated with a much more robust survival advantage in children. ${ }^{12}$ Several longitudinal cooperative group studies have demonstrated that in addition to the favorable response of childhood high-grade gliomas to chemotherapy, a significant correlation exists between the extent of resection and outcome. ${ }^{6,12,50}$ The authors of recent literature reviews focusing on adult patients have failed to demonstrate a similar association..$^{24,30,42}$ Finally, it is becoming clearer that the difference between pediatric and adult high-grade gliomas extends well beyond clinical response to treatment and into the realm of molecular cytogenetics and the pathways that ultimately give rise to the adult and pediatric variants of these tumors. ${ }^{1,4,39,44,47,48} \mathrm{An}$ unfortunate characteristic shared by pediatric and adult high-grade gliomas, however, is their universally poor prognosis: in children with GBM the 5-year survival rates

Abbreviations used in this paper: AA = anaplastic glioma; $\mathrm{CCG}=$ Children's Cancer Group; CNS = central nervous system; $\mathrm{CT}=$ computerized tomography; $\mathrm{GBM}=$ glioblastoma multiforme; $\mathrm{MR}=$ magnetic resonance; NF1 = neurofibromatosis Type 1; $\mathrm{pCV}=$ prednisone; $\mathrm{CCNU}$ (lomustine) vincristine; $\mathrm{PFS}=$ progression free survival; $\mathrm{POG}=$ Pediatric Oncology Group; $\mathrm{QOL}=$ quality of life. range from 5 to $15 \%$, whereas those with AA fare slightly better, with a 20 to $40 \%$ long-term cumulative survival probability. ${ }^{28,35,46}$

The purpose of this brief review is to summarize the epidemiology, clinical presentation, histopathological features, treatment principles, and outcome of pediatric malignant gliomas. The characteristics of childhood and adult high-grade gliomas will be compared and contrasted wherever possible. We will conclude this article with a discussion of some of the recent novel therapeutic strategies.

\section{PEDIATRIC HIGH-GRADE GLIOMAS}

\section{Epidemiological Features}

Precise estimates of the frequency of pediatric highgrade gliomas have been difficult to define, largely because of the tendency in earlier reports to misclassify various low-grade glioma variants under the diagnostic umbrella of high-grade lesions. As such, the validity of many older descriptive and clinical studies of cases reported as high-grade gliomas has been somewhat undermined, simply because of the pathological heterogeneity of the glial tumors that were studied. In more recent descriptive epidemiological studies, however, the authors have begun to clarify incidence and prevalence.

As in adults, the majority of primary supratentorial hemispheric tumors in children are gliomas. In contrast to adults, however, high-grade gliomas are significantly less common than low-grade lesions, comprising only $20 \%$ of all hemispheric gliomas in pediatric series. ${ }^{36}$ Overall, malignant gliomas represent approximately $6.5 \%$ of all newly diagnosed childhood intracranial neoplasms; ${ }^{9,10}$ this 
translates into an incidence of approximately two cases per million children annually.

No review of primary brain tumors, especially those occurring in children, would be complete without a discussion of putative genetic or environmental risk factors. Although clearly beyond the scope of the present discussion, it can safely be said that apart from some wellknown genetic syndromes that predispose to the development of CNS tumors in general, there remains little consensus about the nature and relative magnitude of other risk factors specific to the development of childhood malignant gliomas. ${ }^{51}$

Patients with NF1 harbor a mutation in the neurofibromin gene located on chromosome 17q11.2. Neurofibromin has homology to the RAS-guanosine 5-triphosphatase-activating protein family and normally functions as a tumor suppressor gene. Although the most characteristic intracranial neoplasms in patients harboring this mutation are optic pathway and hypothalamic gliomas, cerebral hemispheric lesions develop in a small percentage of patients. Although low-grade lesions tend to develop in patients with NF1, high-grade tumors have also been reported (Fig. 1). ${ }^{41}$

Turcot syndrome comprises the rare association between colonic polyposis and intracranial neoplasms. This syndrome more than likely represents a collection of distinct disease entities caused by specific germline mutations in a variety of tumor suppressor genes, including the adenomatous polyposis coli gene and DNA mismatch repair genes such as $h M L H 1$ and $h P M S 2 .{ }^{18,34}$ In patients with germline adenomatous polyposis coli mutations there is an increased incidence of medulloblastoma, whereas in those with mismatch repair gene mutations there is generally an increased incidence of gliomas.

Li-Fraumeni cancer syndrome, caused by germline mutations in the $p 53$ tumor suppressor, also predisposes to the development of high-grade gliomas. ${ }^{25}$ A number of less common syndromes have also been linked anecdotal-
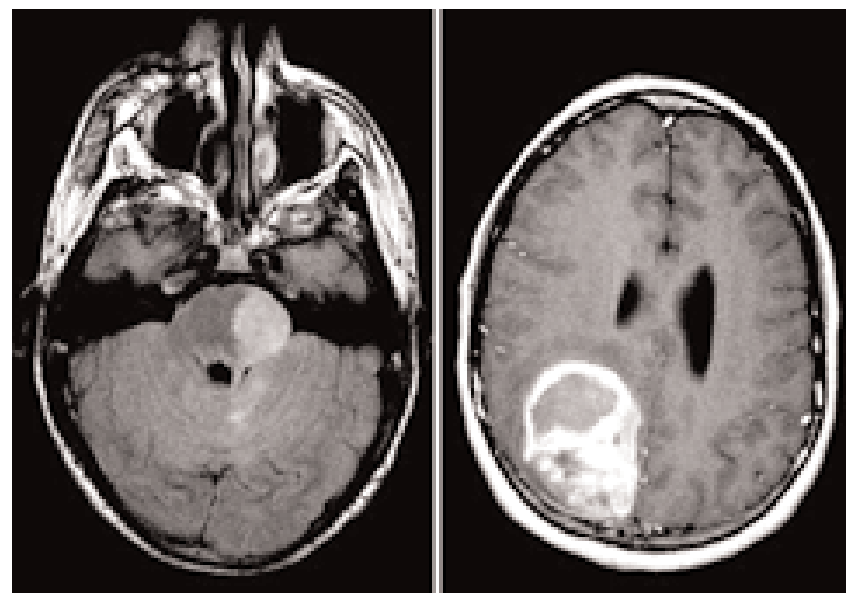

Fig. 1. Axial MR images obtained in a 14-year-old boy with NF1. Left: Image demonstrating brainstem signal change indicative of hamartomatous elements within the lesion. Right: A higher axial image with contrast demonstrating large left parietal hemorrhagic lesion that proved intraoperatively to be a malignant glioma. ly to the development of glial neoplasms, but a molecular basis for these associations has yet to be found.

The one environmental factor known to cause malignant gliomas in childhood is prior irradiation of the brain (Fig. 2). Children who have undergone irradiation in the treatment of prior low-grade gliomas may be particularly susceptible to the transformation of the lesion into a higher grade neoplasm.

\section{Diagnostic Evaluation}

Whereas low-grade gliomas typically present with an insidious onset of symptoms suggestive of intracranial hypertension or a protracted history of seizures, symptoms of high-grade gliomas are typically far more abrupt, with rapidly progressive symptoms or signs of elevated intracranial pressure and/or a focal neurological deficit. ${ }^{3,11}$ Seizures as a mode of presentation of high-grade gliomas are less commonly observed than in the setting of lowgrade tumors (in approximately $30 \%$ of children presenting with malignant gliomas). A significant minority of children, perhaps representing only 5 to $10 \%$ of those with these tumors, present because of a sudden deterioration in neurological status, often as the consequence of intratumoral hemorrhage.

Evidence of disseminated disease at presentation is rare in cases involving high-grade gliomas; ${ }^{16}$ this is in stark contrast to cases involving other malignant pediatric intracranial neoplasms such as supratentorial primitive neuroectodermal tumors.

There is no pathognomonic radiographic appearance that distinguishes the various malignant supratentorial hemispheric tumors from each other. High-grade gliomas typically display irregular or ring enhancement on cranial CT scanning, reflecting the breakdown of the blood-brain barrier or tumor neovascularity. The characteristic MR imaging-documented appearance of malignant gliomas is that of low intensity on $\mathrm{T}_{\text {-weighted sequences; corti- }}$ cal and white matter $\mathrm{T}_{2}$-signal hyperintensity, representing

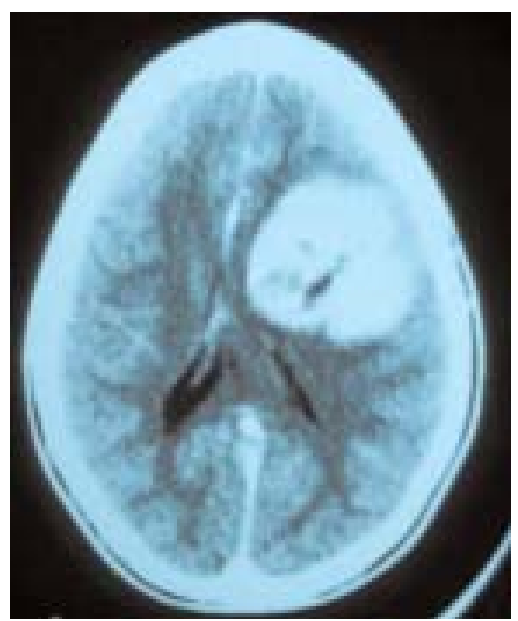

Fig. 2. Axial CT scan revealing left parietal mass lesion in a 12year-old boy who had undergone 5000-rad radiotherapy at age 4 years for a low-grade optic chiasmatic glioma. At age 12 years, the lesion proved to be a malignant glioma and arose in the prior radiation therapy field. 
infiltrating tumor and reactive vasogenic edema, and the aforementioned irregular pattern of contrast enhancement (Fig. 2). Because of the propensity for these lesions to grow rapidly, evidence of significant local mass effect is often seen. The neuroimaging differential diagnosis includes other malignant supratentorial hemispheric tumors, such as supratentorial primitive neuroectodermal tumors, ependymomas, and pleomorphic xanthoastrocytomas. Pleomorphic xanthoastrocytomas, of which approximately $20 \%$ have histological characteristics typical of classical malignant gliomas (see the following section), have a fairly unique neuroimaging appearance in that an enhancing solid component arising near the cortical surface is characteristically observed, in association with an underlying cyst.

Gadolinium-enhanced neuroimaging of the entire neuraxis should be performed only if there is a high index of suspicion for the presence of disseminated disease at the time of initial evaluation or if a competing diagnosis is highly considered in the differential of a child's presenting complaint. Perhaps the best philosophy is to reserve such intensive neuroimaging intervention for the postoperative period, after a firm histopathological diagnosis has been established.

\section{Histopathological Characteristics}

Childhood malignant gliomas may be polar hemispheric tumors or midline, deep-seated lesions; they may be fairly well demarcated from adjacent normal brain or diffusely infiltrative. Grossly, they may show foci of cystic degeneration, hemorrhage, or necrosis.

Supratentorial high-grade gliomas are classically divided into two major subgroups, AA (Grade III) and GBM (Grade IV), based on the presence of characteristic microscopic features. ${ }^{8,23}$ According to the World Health Organization classification scheme, ${ }^{23}$ AAs are characterized by their focal or diffuse hypercellularity, nuclear atypia, nuclear pleomorphism, and the presence of mitotic figures, whereas GBM exhibits vascular proliferation and/or necrosis in addition to the aforementioned features.

Because several less common glial neoplasms also exhibit histological features typical of the classic malignant gliomas (AA and GBM), they are also classified as malignant gliomas in contemporary grading schemes. Grade III oligodendroglial neoplasms have anaplastic features including frequent mitoses, necrosis, and vascular proliferation. Although typically characterized as low-grade, a subset of pleomorphic xanthoastrocytomas exhibit evidence of malignant transformation with necrosis, pronounced mitotic activity, and endothelial proliferation, and are consequently classified as high-grade gliomas. ${ }^{23,27,33}$ Similarly, evidence of increased mitotic activity, necrosis, and endothelial proliferation may be seen in a small proportion of gangliogliomas and juvenile pilocytic astrocytomas. Although these latter lesions undoubtedly display features that would lead to their being labeled as anaplastic under the World Health Organization classification, significant controversy still surrounds the issue of whether these tumors should be grouped with the other high-grade gliomas in view of their more indolent clinical behavior. ${ }^{12}$

The outcome of children with malignant gliomas is highly dependent on tumor histology; in general, patients with GBM fare far worse than those with AA. In the contemporary literature, 5-year survival rates range from 5 to $15 \%$ for children with GBM and from 20 to $40 \%$ for those with AA. ${ }^{28,35,46}$ The authors of several studies have shown that high-grade gliomas other than the predominantly astrocytic AA and GBM are associated with a more favorable outcome. ${ }^{12}$ This may in part be a result of a greater sensitivity of anaplastic oligodendroglial cells in mixed tumors to conventional chemotherapeutic agents, such as the combination regimen of procarbazine, lomustine, and vincristine. $^{5}$

\section{Genetic Alterations and Molecular Pathogenesis}

There are key cytogenetic and molecular differences between adult and pediatric high-grade gliomas, these differences led to the hypothesis that the development of pediatric high-grade gliomas may follow pathways distinct from the traditional primary and secondary malignant glioma paradigm seen in adults. Moreover, a growing link between the molecular characteristics of these tumors and their biological behavior has fueled the search for clinically relevant molecular predictors of outcome.

In comparative genomic hybridization studies investigators have shown that the pattern of chromosomal aberrations differs between pediatric and adult high-grade gliomas, as well as between pediatric AA and pediatric GBM. ${ }^{44}$ Compared with their adult counterparts, childhood high-grade gliomas consistently display more frequent gains of chromosomes $1 \mathrm{p}, 2 \mathrm{q}$, and $21 \mathrm{q}$, as well as more frequent losses of chromosomes $6 \mathrm{q}, 11 \mathrm{q}$, and $16 \mathrm{q}$. Pediatric AAs were typified by changes including $+5 \mathrm{q}$, $-6 \mathrm{q},-9 \mathrm{q},-12 \mathrm{q}$, and $-22 \mathrm{q}$, whereas pediatric GBMs exhibited gains of chromosomes 1q, 3q, and 16p in addition to losses of chromosomes $8 \mathrm{q}$ and $17 \mathrm{p}$. An overwhelming observation, apart from the specific chromosomal aberrations, was that a great deal of chromosomal imbalance exists in pediatric malignant gliomas, with multiple regions of abnormality. There is speculation that a high number of chromosomal aberrations may correlate with an aggressive biological phenotype. ${ }^{44}$

In one of the recent investigations of microsatellite instability in high-grade gliomas, $27 \%$ of pediatric highgrade gliomas compared with $0 \%$ of those in adults displayed length alterations in the tandemly repeated nucleotide sequences $(p<0.001) .{ }^{1}$ Once again, the authors proposed a correlation between microsatellite instability and outcome. ${ }^{1}$

Additional specific molecular alterations have been discovered that appear to have a stronger association with clinical outcome than the aforementioned chromosomal aberrations and evidence of microsatellite instability.

\section{Treatment of Malignant Glioma}

Until recently, the prognosis in children with malignant supratentorial gliomas had been dismal, with most patients dying within 3 years of diagnosis. ${ }^{6,12,35,46}$ Recent aggressive multimodality therapies involving radiotherapy and chemotherapy and founded on a protocol of aggressive resection have been investigated largely under the auspices of multiinstitutional cohorts of pediatric patients with high-grade gliomas (the CCG and POG collaborations); $7,12,17,31,35,46,50$ these investigators have reported more encouraging survival profiles. 


\section{Role of Surgery in Malignant Glioma}

Aggressive resection, with the preservation of neural function, is the cornerstone of the contemporary management of pediatric malignant gliomas (Fig. 3). The goal of surgery is twofold: to obtain tissue for the establishment of an accurate histological diagnosis for the lesion and to achieve safe cytoreduction of the lesion. For reasonably well-circumscribed lesions, a gross-total resection should be the only operative goal, because the extent of tumor removal is positively correlated with long-term survival..$^{50}$ In cases of more infiltrative lesions that prohibit a radical resection, either because they cross the midline or extensively invade critical functional areas of the brain, the goal instead becomes excision of as much tumor as is possible without causing undue neurological morbidity (Fig. 4). Advances in microsurgical technique, in conjunction with the ever-proliferating application of surgical adjuncts including frameless stereotactic guidance systems and other methods for functional mapping of critical brain areas, provide the treating surgeon with a means for the safe removal of tumors previously thought to be unresectable or resectable only with significant morbidity for the patient.

A relationship between the extent of resection and patient survival was first alluded to in the CCG-943 trial, ${ }^{46}$ in which investigators demonstrated improved survival in pediatric patients with GBM, treated with radio- and chemotherapy, in whom some degree of tumor, more substantial than a simple biopsy specimen, had been resected. A more robust conclusion emerged from the subsequent CCG-945 multiinstitutional randomized cohort. ${ }^{12,50}$ The principal aim of the CCG-945 trial was to evaluate the relative efficacy of two alternative chemotherapeutic regimens. The unique nature of this collaborative effort, however, in that data were prospectively recorded with respect to a number of potentially prognostic variables, allowed for the analysts to determine the precise influence of the extent of resection on survival. In children with malignant gliomas undergoing tumor resection of greater than $90 \%$, the 5-year estimate of PFS was $35 \pm 7 \%$, whereas that in children undergoing less than $90 \%$ resection, the 5-year

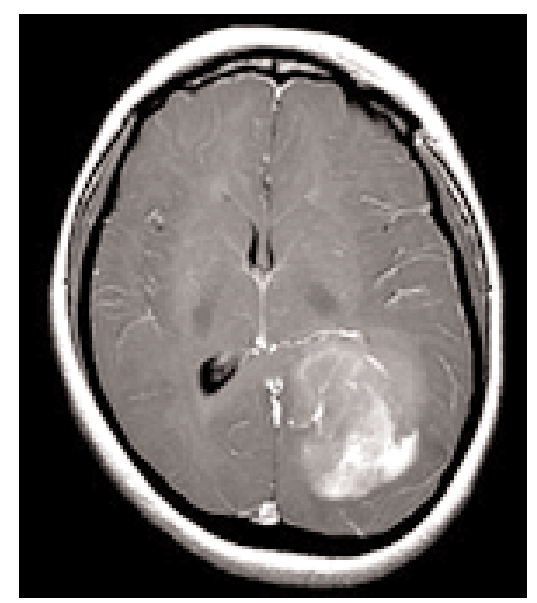

Fig. 3. Axial MR image obtained in a 14-year-old girl with a left occipital high-grade glioma. The lesion enhanced inhomogeneously and was associated with mass effect and some edema.

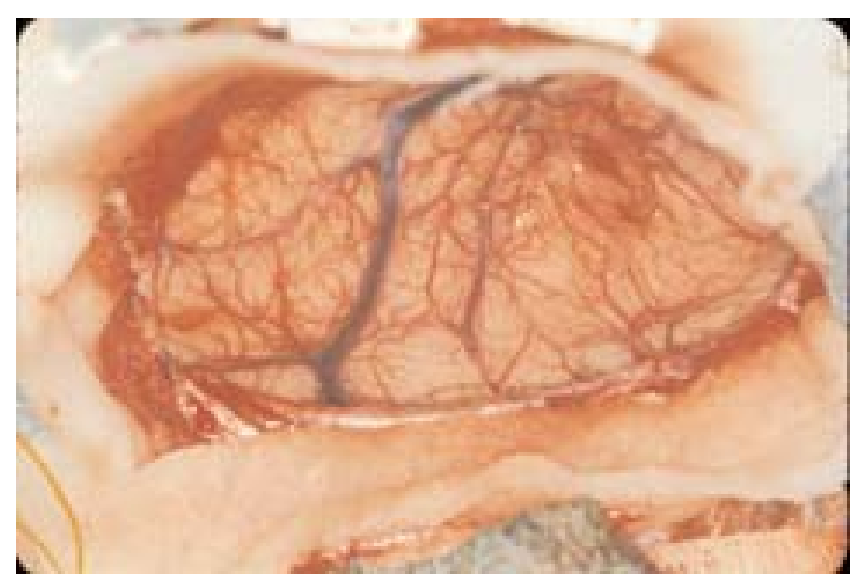

Fig. 4. Intraoperative photograph of high-grade glioma at the cortical surface. Note the variegated appearance of the tumor at the time of surgery.

PFS rate was only $17 \pm 4 \%(p=0.006)$. The strength of the association was demonstrated both in the subgroup of patients with AA ( $>90 \%$ resection $44 \pm 11 \%$ compared with $<90 \%$ resection $22 \pm 6 \%[p=0.055])$ and in the subgroup harboring GBM $(>90 \%$ resection $26 \pm 9 \%$ compared with $<90 \%$ resection $4 \pm 3 \%[\mathrm{p}=0.046]) .{ }^{50} \mathrm{~A}$ caveat involved in interpreting this data is that one must always be aware of the fact that it is impossible to exclude completely the possibility that those tumors possessing more favorable biological characteristics were inherently more amenable to near-complete extirpation. The more favorable outcome in such patients may therefore be partly attributable to these inherent biological characteristics rather than being solely a product of the more aggressive surgical intervention.

\section{Adjuvant Therapy}

Following maximum resection, children with highgrade gliomas routinely undergo radiotherapy targeting the tumor bed and a margin of surrounding brain; the dosing protocol is fairly standard (5000-6000 cGy in 25-30 fractions). Unfortunately, attempts to intensify radiotherapy administration by dose escalation (to a cumulative total dose of $7200 \mathrm{cGy}$ ) in conjunction with hyperfractionation have failed to improve outcome in the setting of highgrade gliomas. 14,32

The use of low-intensity chemotherapy has repeatedly been shown to improve survival over that associated with surgery and radiotherapy alone..$^{12,46}$ The first study to demonstrate this advantage was the CCG- 943 trial, ${ }^{46}$ in which children were randomly allocated to receive radiotherapy followed by the $\mathrm{pCV}$ protocol or radiotherapy alone. In children in the combined radio- and chemotherapy arm the 5-year event-free survival was $46 \%$ compared with that of $18 \%$ in the radiotherapy-alone group. In the CCG945 follow-up study ${ }^{12}$ to the previous trial, investigators tested the superiority of a more complex "eight-drugs-in1-day" regimen over standard pCV chemotherapy in highgrade glioma patients undergoing surgery and radiotherapy. The more elaborate "eight-in-one" protocol did not confer an additional survival benefit over treatment in the 
control arm, probably in large part due to the suboptimum dose intensity of several components of the "eight-in-one" regimen.

Based on these observations, the chemotherapeutic paradigm has shifted from enhancing the complexity of lowintensity regimens, such as the "eight-in-one" protocol used in CCG-945, to dose intensification involving a limited number of agents used in rational combinations. To this end, more intensive submyeloablative regimens have been studied in a neoadjuvant setting, as have myeloablative regimens in an adjuvant role. ${ }^{37}$

In the POG-9135 trial investigators compared the use of neoadjuvant cisplatin/carmustine to cyclophosphamide/ vincristine in patients in whom high-grade gliomas were newly diagnosed; a 20\% 5-year survival rate was observed in the cisplatin/carmustine group as compared with less than 5\% in those treated with cyclophosphamide-vincristine $(\mathrm{p}<0.05)$. In the three-arm CCG-9933 study participants investigated the neoadjuvant administration of three different alkylating agents administered in conjunction with etoposide (carboplatin/etoposide; etoposide/ifosfamide/mesna; and etoposide/cyclophosphamide/mesna) in patients with known postoperative residual disease. Rather than finding a significant beneficial effect of any of these regimens on overall survival, the investigators instead reported an unacceptably high rate of disease progression in all three treatment arms. (Unpublished data.) Similarly, in the POG-9431 study, ${ }^{7}$ designed to compare neoadjuvant procarbazine to topotecan, investigators noted insufficient activity of either agent to warrant further investigation.

In studies involving high-dose chemotherapy in conjunction with stem cell rescue authors have documented promising rates of long-term disease control but at the expense of significant morbidity and mortality as a consequence of the regimens themselves. ${ }^{17,29}$ Similar findings have led many investigators in this area to doubt the value of intensified chemotherapeutic regimens in improving long-term disease control rates, if they indeed appear to arise along with significant deleterious consequences to a child's overall QOL.

For the time being, the $\mathrm{pCV}$ regimen first examined in the CCG-943 and -945 trials appears to be the standard against which other chemotherapeutic protocols will be evaluated in the future, both with respect to efficacy and toxicity profiles.

\section{Prognostic Factors}

Two key prognostic factors with respect to predicting outcome in pediatric high-grade glioma patients have already been discussed. There is a powerful association between the extent of resection and ultimate clinical outcome, as established in the CCG-943 and -945 studies. $^{12,46,50}$ Furthermore, tumor histology is also a very strong predictor of outcome; children with GBM have consistently been shown to fare worse than those with AA who have been matched with respect to other prognostic variables. ${ }^{12,46}$ Despite these significant associations, however, the aforementioned clinical and histopathological features fail to predict adequately outcomes in children with malignant gliomas, indicating a need to identify relevant molecular markers of clinical tumor behavior that will guide subsequent therapeutic decision making. As noted previously, a number of cytogenetic and molecular factors have been associated with outcome; the two that will be discussed in greater detail here include p53 expression status ${ }^{38,39}$ and tumor proliferation index. ${ }^{40}$

In the CCG substudy B975 investigators were able to obtain tumor specimens in 170 of the 231 children enrolled in the larger CCG-945 study, making this collection of pathological specimens representing pediatric highgrade gliomas the largest ever assembled. Alterations in the p53 pathway were examined in this cohort. In children with tumors that displayed evidence of $\mathrm{p} 53$ overexpression and/or TP53 mutations, a significantly worse PFS was demonstrated than in those with tumors that lacked these features. The 5-year PFS rate was $44 \pm 6 \%$ in the 74 children in whom there were low levels of $\mathrm{p} 53$ expression compared with $17 \pm 6 \%$ in 41 patients with p53 overexpression $(\mathrm{p}=0.0001) \cdot{ }^{39}$ Additionally, it was found that abnormalities of p53 expression were far more common in GBMs $(58 \%)$ than in AAs $(26 \%)$ or other Grade III gliomas $(\mathrm{p}<0.002)$. Despite the strong association between histology and p53 alterations, p53 expression status was found to be an independent predictor of outcome for each histological stratum $(\mathrm{p}=0.005) .{ }^{39}$ Furthermore, an association was found between $\mathrm{p} 53$ expression status and patient age; in children younger than 3 years of age there was a significantly lower frequency of TP53 mutation than in older children..$^{38}$ This finding has led to the suggestion that malignant gliomas in younger patients may arise via a molecular pathway not involving p53.

Proliferation index, as assessed by MIB-1 antibody labeling of the nuclear Ki-67 antigen, was also found to correlate highly with patient outcome. Ninety-eight tumors obtained in the larger CCG-945 study were assessed using this method. The 5-year PFS rate was $33 \pm 7 \%$ in 43 patients in whom MIB-1 labeling indices were less than $18 \%, 22 \pm 8 \%$ in the 27 in whom labeling indices were between 18 and $36 \%$, and $11 \pm 6 \%$ in the 28 patients in whom labeling indices were greater than $36 \%(\mathrm{p}=$ $0.003) .{ }^{40}$ Consistent with the experience in those studies aimed at ascertaining p53 marker status was the finding that the MIB-1 labeling index was also significantly correlated with tumor histology. Nevertheless, the link between MIB-1 labeling index and outcome was a robust one: the significance of the association held even after the independent effects of tumor histology on outcome were accounted for $(p=0.004)$. Although the finding that tumors with a high proliferative activity as assessed by this method tended to be more biologically aggressive is not terribly surprising, it does point toward the need for a better understanding of the cell cycle so that it may be appropriately targeted in the search for novel therapeutic strategies aimed at controlling these notoriously aggressive tumors.

In addition to $\mathrm{p} 53$ expression status and proliferative index, a variety of other molecular markers are currently under intense investigation for their potential association with outcome in childhood malignant gliomas. Additionally, it is hoped that some of these markers will ultimately prove useful as a means for prospectively identifying those children in whom a given therapeutic approach would yield a favorable response, thus serving as a method for pretreatment "risk stratification". ${ }^{37}$ 
The starting point for many of these studies arose from the well-documented association of certain molecular markers with disease progression in the setting of adult malignant gliomas. Epidermal growth factor receptor amplification, ${ }^{4,47}$ PTEN (phosphatase and tensin homolog detected on chromosome 10) deletions, ${ }^{43}$ and alterations in specific cell cycle control genes including p14 $4^{A R F 21}$ are some representative examples of the pathways currently being studied.

It is fitting to conclude this section by reiterating a point so eloquently made by Pollack and colleagues: ${ }^{39,40}$ no marker has yet been able to identify a subset of pediatric malignant gliomas that have a truly "favorable" prognosis, as even among the subgroups of patients whose tumors possess "favorable" marker profiles, more than $60 \%$ eventually die of disease progression, despite provision of the best available conventional treatments.

\section{Tumor Recurrence}

At the time one first suspects tumor recurrence in a patient, a complete evaluation for the determination of relapse is warranted. Although pediatric malignant gliomas typically recur at the primary site, they may also spread via the subarachnoid space to noncontiguous CNS sites. Leptomeningeal dissemination has been observed in a small proportion of children with high-grade gliomas. ${ }^{11}$ Metastases outside the CNS have been reported but are extremely rare.

Often, a recurrence at the primary site may be amenable to additional locoregional therapy including resection or stereotactic radiosurgery. Experimental chemotherapeutic protocols may also be considered if local therapy is not feasible. Any further intervention, however, must be individualized on the basis of the initial tumor type, the length of time between initial treatment and recurrence of the lesion, and the overall clinical picture. Despite aggressive therapy aimed at controlling recurrent disease, the prognosis for long-term survival is not good. In the CCG-945 trial, ${ }^{12,50}$ nearly all children in whom recurrence developed after initial surgery, radiotherapy, and chemotherapy died within 1 year. In those who received no additional treatment, mean survival was less than 2 months; in those children who were in sufficiently good clinical condition to warrant further treatment, the mean survival despite additional conventional chemotherapy, with or without further surgery, was less than 9 months.

In the setting of recurrent disease, high-dose myeloablative chemotherapy (thiotepa combined with etoposide) and autologous stem cell rescue yielded a 4-year eventfree survival rate of $22 \pm 7 \%$ compared with $2 \pm 1 \%$ in children in the CCG-945 study who underwent conventional chemotherapy at the time of recurrence. ${ }^{13}$ The survival advantage associated with this high-dose regimen applied in both patients with GBM $(14 \pm 8 \%$ in patients on the experimental high-dose regimen compared with $0 \%$ in those on conventional chemotherapy [p $=0.003]$ ) and for those with AA $(36 \pm 13 \%$ in patients on the experimental high-dose regimen compared with $10 \pm 5 \%$ in those on conventional chemotherapy, $[\mathrm{p}=0.036])$. The benefit of high dose chemotherapy with stem cell rescue, however, was observed only in patients receiving this treatment in whom minimal tumor burden was demonstrated following radical resection.
In terms of tumor recurrence, improvements in patient outcome will be dependent on implementing novel therapeutic approaches targeting the molecular pathways that are aberrantly activated in these tumors, finding new mechanisms to improve the potency of locally delivered therapies, and modifying the host immune response against the tumor by using vaccines and immunomodulatory therapies.

\section{Outcome and Prognosis}

Despite refinements in the multimodality management of patients with these tumors, the overall long-term survival probability for children with supratentorial malignant gliomas remains dismal, with PFS beyond 5 years occurring in fewer than $30 \%$. The outcome in children with high-grade gliomas is generally more favorable than in adults, but it is uncertain whether this difference is caused by variations in tumor biology, the therapeutic strategies used, inherent tumor resectability, or other factors remaining to be elucidated.

In contemporary studies of novel therapeutic strategies there are two principal goals. The first is to develop new protocols designed to improve overall survival (some of the more promising of these new ventures are discussed in the subsequent section). The second, which should not be considered a secondary objective in any sense, involves reducing the morbidity associated with conventional therapy, thereby preserving a child's QOL during the treatment phase.

A surgeon must be judicious when undertaking the resection of a malignant glioma; any potential envisioned gains arising from a more radical resection must be balanced against the chances of causing real neurological harm to the patient.

The routine use of radiotherapy in the overall management of pediatric high-grade gliomas is associated with the well-known risks of delayed cognitive and neuropsychological sequelae. Although these patients are typically not treated with whole-brain irradiation, the radiation fields required for optimum treatment of these lesions quite commonly encompass a significant territory of brain tissue, thereby subjecting patients to a similar risk of adverse neurocognitive consequences. Protocols are being evaluated in which the aim is to target more precisely the neoplastic tissue while minimizing the volume of normal brain irradiated. Moreover, in light of the often devastating consequences of cranial irradiation in very young children, considerable attention has been devoted to devising strategies that either delay or avoid radiotherapy entirely in this population, by first administering an extended course of intensive chemotherapy.

Neoadjuvant and adjuvant chemotherapeutic protocols subject patients to significant toxicity involving the hematopoietic system in particular but also to other general body systems. Conventional low-intensity regimens appear to be relatively well tolerated, but, as previously mentioned, high-dose myeloablative and submyeloablative regimens are associated with substantial morbidity and mortality rates. ${ }^{17,29}$ Clearly, such significant toxicityrelated considerations must be taken into account when discussing potential alternative therapeutic strategies with a child's caregivers. Suffice it to say that ongoing study is required to determine fully whether such novel approach- 
es truly translate into meaningful improvements in overall survival and QOL.

\section{Future Directions}

The efficacy of surgery and radiotherapy in the management of pediatric supratentorial high-grade gliomas has been established. The results obtained in several of the cooperative group studies discussed in this review have supported the finding that the provision of adjuvant chemotherapy in addition to irradiation improves the probability of long-term event-free survival. ${ }^{12,46}$ As suggested by the proliferation of chemotherapeutic trials in recent years, however, the optimal treatment regimen for pediatric malignant gliomas has yet to be established.

It is anticipated that further gains in survival rates will be yielded by investigation of several novel management approaches. One field of intense investigation is the study of novel chemotherapeutic agents such as antiangiogenic factors targeted at the hypervascular phenotype of malignant gliomas ${ }^{49}$ or pharmacological approaches designed to interfere with the signal transduction pathways gone awry in these lesions; potential investigational agents in this regard include epidermal growth factor receptor inhibitors ${ }^{19}$ and platelet-derived growth factor receptor inhibitors. ${ }^{22}$ Additional studies are being conducted to examine the effect of new combinations of drugs, optimization of chemotherapy dose intensity, and chemotherapeutic measures designed to enhance the efficacy of conventional radiotherapy ("radiosensitizing" agents). Finally, new systems designed to enhance drug delivery to local sites are also at the forefront of research. Representative tactics include the following: antibody- or ligandmediated targeting of tumor cell antigens with the intent of inciting a host immune response against the tumor, ${ }^{20}$ or delivery of a cytotoxic agent ${ }^{26}$ or radionuclide conjugate ${ }^{45}$ directly to the tumor; gene therapy involving toxin-producing viral vector constructs to induce selective killing of rapidly proliferating tumor cells; ${ }^{2}$ and cytokine-producing constructs designed to induce an active host immune response against the tumor. ${ }^{15}$ The practice of such studies will, of necessity, be predicated on the central, blinded review of pathological specimens so that direct comparisons between different study results can be made. It appears that the current standard against which these novel investigational agents will be compared will be the $\mathrm{pCV}$ protocol examined under the auspices of the CCG-945 multiinstitutional cohort.

\section{CONCLUSIONS}

Ongoing delineation of the basic molecular mechanisms at work in pediatric supratentorial high-grade gliomas will serve to fuel the search for specific targeted therapies directed against these very aggressive lesions. Furthermore, continual refinements to the contemporary multimodality treatment of these tumors are being made. Taken together, these advances set the stage for improving the future prospects of children in whom these malignant supratentorial gliomas are diagnosed, both with respect to survival and QOL.

\section{References}

1. Alonso M, Hamelin R, Kim M, et al: Microsatellite instability occurs in distinct subtypes of pediatric but not adult central nervous system tumors. Cancer Res 61:2124-2128, 2001

2. Andreansky S, Soroceanu L, Flotte ER, et al: Evaluation of genetically engineered herpes simplex viruses as oncolytic agents for human malignant brain tumors. Cancer Res 57:1502-1509, 1997

3. Artico M, Cervoni L, Celli P, et al: Supratentorial glioblastoma in children: a series of 27 surgically treated cases. Childs Nerv Syst 9:7-9, 1993

4. Bredel M, Pollack IF, Hamilton RL, et al: Epidermal growth factor receptor expression and gene amplification in high-grade non-brainstem gliomas of childhood. Clin Cancer Res 5: 1786-1792, 1999

5. Cairncross JG, Macdonald DR, Ramsay DA: Aggressive oligodendroglioma: a chemosensitive tumor. Neurosurgery 31: 78-82, 1992

6. Campbell JW, Pollack IF, Martinez AJ, et al: High-grade astrocytomas in children: Radiologically complete resection is associated with an excellent long-term prognosis. Neurosurgery 38:258-264, 1996

7. Chintagumpala M, Burger P, McCluggage C, et al: DNA mismatch repair and $\mathrm{O}^{6}$-alkylguanine-DNA alkyltransferase (AGT) analysis and response to procarbazine in malignant glioma in children-a Pediatric Oncology Group (POG) study. Med Pediatr Oncol 33:159, 1999 (Abstract)

8. Daumas-Duport C, Scheithauer B, O'Fallon J, et al: Grading of astrocytomas. A simple and reproducible grading method. Cancer 62:2152-2165, 1988

9. Dohrmann GJ, Farwell JR, Flannery JT: Astrocytomas in childhood: a population-based study. Surg Neurol 23:64-68, 1985

10. Dohrmann GJ, Farwell JR, Flannery JT: Glioblastoma multiforme in children. J Neurosurg 44:442-448, 1976

11. Dropcho EJ, Wisoff JH, Walker RW, et al: Supratentorial malignant gliomas in childhood: a review of fifty cases. Ann Neurol 22:355-364, 1987

12. Finlay JL, Boyett JM, Yates AJ, et al: Randomized phase III trial in childhood high-grade astrocytoma comparing vincristine, lomustine, and prednisone with the eight-drugs-in-1-day regimen. Children's Cancer Group. J Clin Oncol 13: 112-123, 1995

13. Finlay JL, Goldman S, Wong MC, et al: A pilot study of high dose thiotepa and etoposide with autologous bone marrow rescue in children and young adults with recurrent CNS tumors. The Children's Cancer Group. J Clin Oncol 14:2495-2503, 1996

14. Fulton DS, Urtasun DC, Scott-Brown I, et al: Increasing radiation dose intensity using hyperfractionation in patients with malignant glioma. Final report of a prospective phase I-II dose response study. J Neuro-oncol 14:63-72, 1992

15. Glick RP, Lichtor T, Mogharbel A, et al: Intracerebral versus subcutaneous immunization with allogenic fibroblasts genetically engineered to secrete interleukin- 2 in the treatment of central nervous system glioma and melanoma. Neurosurgery 41: 898-907, 1997

16. Grabb PA, Albright AL, Pang D: Dissemination of supratentorial malignant gliomas via the cerebrospinal fluid in children. Neurosurgery 30:64-71, 1992

17. Grovas AC, Boyett JM, Lindsley K, et al: Regimen-related toxicity of myeloablative chemotherapy with BCNU, thiotepa, and etoposide followed by autologous stem cell rescue for children with newly diagnosed glioblastoma multiforme: report from the Children's Cancer Group. Med Pediatr Oncol 33:83-87, 1999

18. Hamilton SR, Lui B, Parsons RE, et al: The molecular basis of Turcot's syndrome. N Engl J Med 332:839-847, 1995

19. Heimberger AB, Learn CA, Archer GE, et al: Brain tumors in mice are susceptible to blockade of epidermal growth factor receptor (EGFR) with the oral, specific, EGFR-tyrosine kinase inhibitor ZD1839 (IRESSA). Clin Cancer Res 8:3496-3502, 2002 
20. Hills D, Rowlinson-Busza G, Gullick WJ: Specific targeting of a mutant, activated FGF receptor found in glioblastoma using a monoclonal antibody. Int J Cancer 63:537-543, 1995

21. Ichimura K, Bolin MB, Goike HM, et al: Deregulation of the $\mathrm{p} 14 \mathrm{ARF} / \mathrm{MDM} 2 / \mathrm{p} 53$ pathway is a prerequisite for human astrocytic gliomas with G1-s transition control gene abnormalities. Cancer Res 60:417-424, 2000

22. Kilic T, Alberta JA, Zdunek PR, et al: Intracranial inhibition of platelet-derived growth factor-mediated glioblastoma cell growth by an orally active kinase inhibitor of the 2-phenylaminopyrimidine class. Cancer Res 60:5143-5150, 2000

23. Kleihues P, Burger PC, Scheithauer BW (eds): Histological Typing of Tumors of the Central Nervous System, ed 2. Berlin: Springer-Verlag, 1993

24. Kreth FW, Warnke PC, Scheremet R et al: Surgical resection and radiation therapy versus biopsy and radiation therapy in the treatment of glioblastoma multiforme. J Neurosurg 78: 762-766, 1993

25. Kyritsis AP, Bondy ML, Xiao M, et al: Germline p53 gene mutations in subsets of glioma patients. J Natl Cancer Inst 86:344-349, 1994

26. Laske DW, Youle RJ, Oldfield EH: Tumor regression with regional distribution of targeted toxin TF-CRM107 in patients with malignant brain tumors. Nat Med 3:1362-1368, 1997

27. Macaulay RJ, Jay V, Hoffman HJ, et al: Increased mitotic activity as a negative prognostic indicator in pleomorphic xanthoastrocytoma. Case report. J Neurosurg 79:761-768, 1993

28. Marchese MJ, Chang CH: Malignant astrocytic gliomas in children. Cancer 65:2771-2778, 1990

29. Mason WP, Grovas A, Halpern S, et al: Intensive chemotherapy and bone marrow rescue for young children with newly diagnosed malignant brain tumors. J Clin Oncol 16:210-221, 1998

30. Nazzaro JM, Neuwelt EA: The role of surgery in the management of supratentorial intermediate and high-grade astrocytomas in adults. J Neurosurg 73:331-344, 1990

31. Nicholson HS, Krailo M, Ames MM, et al: Phase I study of temozolomide in children and adolescents with recurrent solid tumors: a report from the Children's Cancer Group. J Clin Oncol 16:3037-3043, 1998

32. Packer RJ, Boyett JM, Zimmerman RA, et al: Hyperfractionated radiation therapy ( $72 \mathrm{~Gy}$ ) for children with brainstem gliomas. A Children's Cancer Group Phase I/II Trial. Cancer 72:1414-1421, 1993

33. Pahapill PA, Ramsay DA, Del Maestro RF: Pleomorphic xanthoastrocytoma: case report and analysis of the literature concerning the efficacy of resection and the significance of necrosis. Neurosurgery 38:822-829, 1996

34. Paraf F, Jothy S, VanMeir EG: Brain tumor-polyposis syndrome: two genetic diseases? J Clin Oncol 15:2744-2758, 1997

35. Phuphanich S, Edwards MS, Levin VA, et al: Supratentorial malignant gliomas of childhood. Results of treatment with radiation therapy and chemotherapy. J Neurosurg 60:495-499, 1984

36. Pollack IF: Brain tumors in children. N Engl J Med 331: 1500-1507, 1994

37. Pollack IF, Boyett JM, Finlay JL: Chemotherapy for high-grade gliomas of childhood. Childs Nerv Syst 15:529-544, 1999
38. Pollack IF, Finkelstein SD, Burnham J, et al: Age and TP53 mutation frequency in childhood gliomas: results in a multiinstitutional cohort. Cancer Res 61:7404-7407, 2001

39. Pollack IF, Finkelstein SD, Woods J, et al: Expression of p53 and prognosis in childhood malignant gliomas. N Engl J Med 346:420-427, 2002

40. Pollack IF, Hamilton RL, Burnham J, et al: The impact of proliferation index on outcome in childhood malignant gliomas: results in a multi-institutional cohort. Neurosurgery 50: 1238-1245, 2002

41. Pollack IF, Mulvihill JJ: Special issues in the management of gliomas in children with neurofibromatosis 1 . J Neurooncol 28:257-268, 1996

42. Quigley MR, Maroon JC: The relationship between survival and the extent of the resection in patients with supratentorial malignant gliomas. Neurosurgery 29:385-389, 1991

43. Raffel C, Frederick L, O'Fallon JR, et al: Analysis of oncogene and tumor suppressor gene alterations in pediatric malignant astrocytomas reveals reduced survival for patients with PTEN mutations. Clin Cancer Res 5:4085-4090, 1999

44. Rickert $\mathrm{CH}$, Sträter R, Kaatsch $\mathrm{P}$, et al: Pediatric high-grade astrocytomas show chromosomal imbalances distinct from adult cases. Am J Pathol 158:1525-1532, 2001

45. Schold SC Jr, Zalutsky MR, Coleman RE, et al: Distribution and dosimetry of I-123-labeled monoclonal antibody 81C6 in patients with anaplastic glioma. Invest Radiol 28:488-496, 1993

46. Sposto R, Ertel IJ, Jenkin RD, et al: The effectiveness of chemotherapy for treatment of high grade astrocytoma in children: results of a randomized trial. A report from the Childrens Cancer Study Group. J Neurooncol 7:165-177, 1989

47. Sung T, Miller DC, Hayes RL, et al: Preferential inactivation of the p53 tumor suppressor pathway and lack of EGFR amplification distinguish de novo high grade pediatric astrocytomas from de novo adult astrocytomas. Brain Pathol 10:249-259, 2000

48. Sure U, Ruedi D, Tachibana O, et al: Determination of p53 mutations, EGFR overexpression, and loss of p16 expression in pediatric glioblastomas. J Neuropathol Exp Neurol 56: 782-789, 1997

49. Takamiya Y, Brem H, Ojeifo J, et al: AGM-1470 inhibits the growth of human glioblastoma cells in vitro and in vivo. Neurosurgery 34:869-875, 1994

50. Wisoff JH, Boyett JM, Berger MS, et al: Current neurosurgical management and the impact of the extent of resection in the treatment of malignant gliomas of childhood: a report of the Children's Cancer Group trial no. CCG-945. J Neurosurg 89: 52-59, 1998

51. Wrensch M, Minn Y, Chew T, et al: Epidemiology of primary brain tumors: current concepts and review of the literature. Neuro-oncol 4:278-299, 2002

Manuscript received December 23, 2002.

Accepted in final form January 22, 2003.

Address reprint requests to: James T. Rutka, M.D., Ph.D., F.R.C.S.(C), Division of Neurosurgery, The Hospital for Sick Children, Suite 1504, 555 University Avenue, Toronto, Ontario, M5G 1X8, Canada. email: james.rutka@sickkids.ca. 